\title{
ERGONOMICS SPECIFICATIONS AND DESIGN OF A HMI FOR AN INFORMATIONAL SAFE DRIVING SUPPORT SYSTEM
}

\author{
Laurent Nicolas \\ Perception and Human Factors \\ Division of Research and Automobile Innovation \\ PSA Peugeot Citroën \\ Vélizy, FRANCE \\ E-mail: nicola30@mpsa.com
}

\begin{abstract}
Summary : This paper describes the first ergonomics step for the design of a HMI for an informational safe driving support system. The objectives of the experimentation presented below, are to supply first settings corresponding to an alert and to collect first "subjectives" evaluations of the HMI tested with the aim of a final evaluation and of recommendations for the conception.
\end{abstract}

\section{INTRODUCTION AND OBJECTIVES}

A research division of PSA Peugeot Citroën is actually developing an informational safe driving support system. Our ergonomics division has the responsibility of the HMI design of this project. The system aims at alerting the driver in case of a dangerous situation.

In this context, two goals were identified for the ergonomics study:

- Define and specify the alerting HMI for different modalities;

- Evaluate the global system in driving situations.

\section{EXPERIMENTATION}

To begin, we conducted a first on-road experiment, with HMI but without the final system. The objective was to make a panel of drivers specify which HMI settings correspond to good alerts for the system and justify why.

\section{The HMI prototyped}

Visual, auditive and haptic modalities were considered to provide this alert. As shown on the figure below, five HMI were studied:

- A sound, provided by speakers integrated in the driver headrest;

- Two flashing lights down the windscreen;

- Two flashing arrows in the instrument cluster;

- A vibrator in the steering wheel;

- Two vibrators in the driver seat. 




Figure 1 : Prototyped and tested HMI

\section{Protocol}

The observer controlled one parameter for each HMI with an embedded computer (e.g. sound volume, voltage, flashing frequency). By following a "M" protocol (see Figure 2), people were asked to specify which settings could be an alert. We did not ask taste opinions but efficiency assessments. At the end of the driving, an interview was conducted by the observer, with a support questionnaire, to evaluate the HMI.

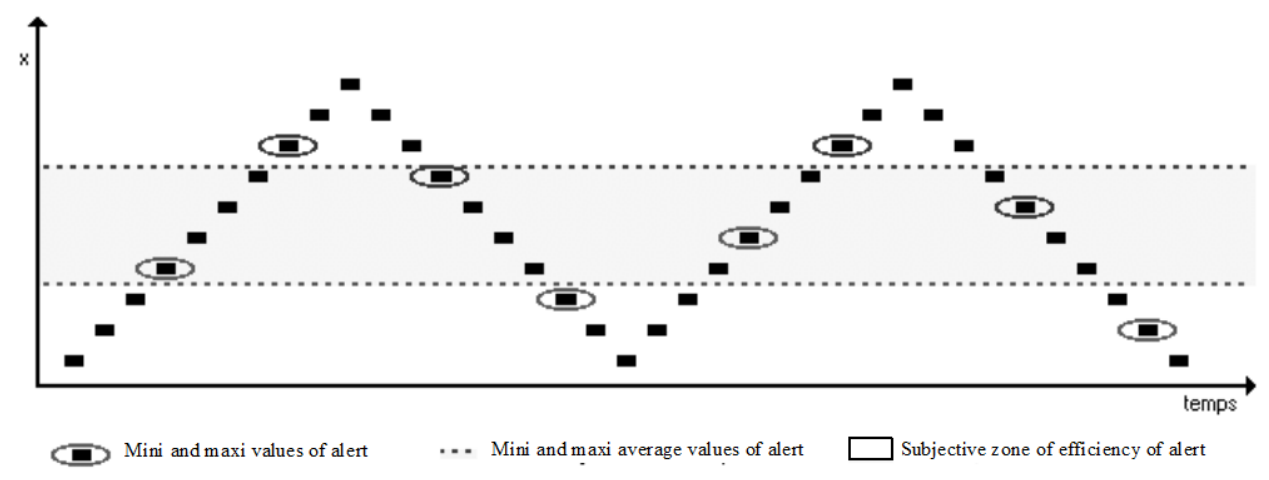

Figure 2 : "M” protocol - one trial increasing the parameter followed by a second decreasing it, this for twice

\section{RESULTS AND PERSPECTIVES}

Two types of data were processed. We calculated the average "alert settings" and we choose the mode, for each HMI, as a final specification. 
We also analyzed the verbal protocols collected during the interviews. A content analysis determined seven ergonomics criteria, which people evaluated the HMI with:

- Attraction;

- Alert level;

- Representation of nature of danger;

- Localization of the danger;

- Incitement to act;

- Comfort;

- "Distinguishability”.

The verbal analysis put forward the advantages and defaults of each HMI (e.g. attraction of the sound, alert level of the flashing lights, very bad attraction of the instrument cluster arrows). These first results allowed us to make choices for the second part of the project (e.g. leaving of one HMI, connecting of vibrations and lights).

In this second part, we will evaluate the global system, its function and remaining HMI, in real driving situation. For this, two other experiments are scheduled.

The first is a "long term use" evaluation. A very few people (2 or 3) will use the system during one month. We will conduct weekly interviews, to evaluate the usability and the use of the system and the ergonomics characteristics of its HMI. In complement, the second experiment will be a secondary-task scenario based evaluation. 